\title{
Electronic structure and magnetic properties of selected lanthanide and actinide intermetallic Laves-phase alloys
}

\author{
Eriksson, Olle; Johansson, Börje; Brooks, M. S. S.; Skriver, Hans Lomholt
}

Published in:

Physical Review B

Link to article, DOI:

10.1103/PhysRevB.40.9519

Publication date:

1989

Document Version

Publisher's PDF, also known as Version of record

Link back to DTU Orbit

Citation (APA):

Eriksson, O., Johansson, B., Brooks, M. S. S., \& Skriver, H. L. (1989). Electronic structure and magnetic properties of selected lanthanide and actinide intermetallic Laves-phase alloys. Physical Review B, 40(14), 9519-9528. https://doi.org/10.1103/PhysRevB.40.9519

\section{General rights}

Copyright and moral rights for the publications made accessible in the public portal are retained by the authors and/or other copyright owners and it is a condition of accessing publications that users recognise and abide by the legal requirements associated with these rights.

- Users may download and print one copy of any publication from the public portal for the purpose of private study or research.

- You may not further distribute the material or use it for any profit-making activity or commercial gain

- You may freely distribute the URL identifying the publication in the public portal 


\title{
Electronic structure and magnetic properties of selected lanthanide and actinide intermetallic Laves-phase alloys
}

\author{
Olle Eriksson* and Börje Johansson \\ Condensed Matter Theory Group, Department of Physics, University of Uppsala, P.O. Box 530, S-75121 Uppsala, Sweden \\ M. S. S. Brooks \\ European Institute for Transuranium Elements, P.O. Box 2340, D-7500 Karlsruhe, Federal Republic of Germany \\ and Condensed Matter Theory Group, Department of Physics, University of Uppsala, P.O. Box 530, S-751 21 Uppsala, Sweden \\ H. L. Skriver \\ Laboratory of Applied Physics, Technical University of Denmark, DK-2800 Lyngby, Denmark
}

(Received 4 April 1989)

\begin{abstract}
The electronic structure and magnetic properties of some yttrium and uranium Laves-phase pseudobinary alloys with $3 d$ elements have been calculated. The calculations were done by simulating the electronic structure of the alloy by that of an ordered compound with the same stoichiometry. In general a good agreement between the experimental and theoretical magnetic moment was found, indicating that the spurious long-range order of the calculations is of minor importance. A comparison between the present supercell cluster approach and the virtual-crystal approximation for the electronic structure and cohesive properties is presented for $\mathrm{Y}\left(\mathrm{Fe}_{0.75} \mathrm{Co}_{0.25}\right)_{2}$ and $\mathrm{U}\left(\mathrm{Fe}_{0.5} \mathrm{Ni}_{0.5}\right)_{2}$.
\end{abstract}

\section{INTRODUCTION}

The intermetallic compounds between rare earths or actinides and $d$ transition elements have a variety of intriguing properties. For example, equilibrium volume, crystal structure, and transport and magnetic properties exhibit a vast variation for the various compounds. ${ }^{1}$ One of these large (in terms of existing materials) classes of compounds is the group of systems crystallizing in the Laves-phase structure $(C 15, C 14)$. Both the rare earths and the actinide elements form Laves-phase compounds with $3 d$ transition elements, ${ }^{1}$ and in the present work we will discuss a subgroup of these systems. As prototypes for the rare-earth and actinide behavior in these materials we have chosen yttrium and uranium Laves-phase compounds, respectively. Thus we will study properties in relation to the $\mathrm{UM}_{2}(M=\mathrm{Mn}, \mathrm{Fe}, \mathrm{Co}$, and $\mathrm{Ni})$ systems, where $\mathrm{UMn}_{2}$ ( $C 15$ structure) and $\mathrm{UCo}_{2}$ ( $C 15$ structure) have been reported to have a paramagnetic susceptibility whereas $\mathrm{UFe}_{2}$ (C15 structure) and $\mathrm{UNi}_{2}$ (C14 structure) are ferromagnets. ${ }^{2}$ Detailed calculations for these $C 15$ systems have been performed previously, where especially the magnetic ground-state properties were emphasized. ${ }^{3}$ Yttrium, which is a $4 d$ transition element, is due to its number of valence electrons and chemical behavior referred to as a member of the rare-earth series and, like many of the lanthanides, forms cubic Laves-phase compounds with $\mathrm{Mn}, \mathrm{Fe}$, Co and $\mathrm{Ni}^{1}$ The study of yttrium in these compounds has the advantage that no local $f$ moments have to be considered, and therefore an understanding of the magnetic behavior is less complex than for the lanthanides with magnetic $4 f$ electrons. Experimentally $\mathrm{YNi}_{2}$ is known to be a weak paramagnet, $\mathrm{YCo}_{2}$ an exchange-enhanced paramagnet, $\mathrm{YFe}_{2}$ a ferromagnet and, finally, $\mathrm{YMn}_{2}$ an antiferromagnet. ${ }^{4}$ Several authors have predicted that $\mathrm{YCo}_{2}$ should undergo a metamagnetic transition in a magnetic field of the order of $100 \mathrm{~T}^{5}$ Calculations ${ }^{6}$ of the magnetic moment for $\mathrm{YFe}_{2}$ have been found to agree well with experiment. ${ }^{7}$ From the experimental facts one notices that there is a striking similarity between the uranium and yttrium series, e.g., the Laves-phase compounds with $\mathrm{Fe}$ are magnetic whereas the compounds with Co are nonmagnetic. However, this similarity does not hold for the $\mathrm{Ni}$ systems and an explanation for this different behavior has been proposed where the magnetism in $\mathrm{UNi}_{2}$ is believed to originate from the $5 f$ electrons. ${ }^{8}$ Recently, it has been shown that a theoretical treatment with delocalized $U$ $5 f$ electrons accounts very well for the equilibrium volumes as well as the magnetic properties for the $\mathrm{UM}_{2}(M=\mathrm{Mn}, \mathrm{Fe}, \mathrm{Co}$, and $\mathrm{Ni}$ ) systems. ${ }^{3}$ This supports the view of itinerant $5 f$ electrons in these compounds.

For $\mathrm{UFe}_{2}$ the coefficient of the linear contribution to the specific heat $\gamma$ has been measured to be as high as 55 $\mathrm{mJ} / \mathrm{mol} \mathrm{K}^{2}$ (Refs. 2 and 9) which strongly suggests that there are itinerant electrons in narrow bands at the Fermi level. Similar high- $\gamma$ values have also been found for $\mathrm{UMn}_{2}, \mathrm{UCO}_{2}$, and $\mathrm{UNi}_{2}$. The delocalized picture of the $5 f$ electrons in the $\mathrm{UM}_{2}(M=\mathrm{Mn}, \mathrm{Fe}, \mathrm{Co}$, and $\mathrm{Ni})$ systems is also supported by other experiments, like, for example, photoelectron spectroscopy where a pronounced peak, identified as a $5 f$ signal, pinned to the Fermi level has been observed for all these uranium systems. ${ }^{10}$

Recently the magnetic properties of a variety of pseudobinary compounds $A\left(M_{1-x} M_{x}^{\prime}\right)_{2}$ have been reported. This includes, for example, the following systems: $\mathrm{U}\left(\mathrm{Fe}_{1-x} \mathrm{Ni}_{x}\right)_{2}, \quad \mathrm{U}\left(\mathrm{Fe}_{1-x} \mathrm{Mn}_{x}\right)_{2}, \quad \mathrm{U}\left(\mathrm{Fe}_{1-x} \mathrm{Cr}_{x}\right)_{2}, \quad$ and $\mathrm{Y}\left(\mathrm{Fe}_{1-x} \mathrm{Co}_{x}\right)_{2} \cdot{ }^{4}$ Such a systematic experimental study of 
magnetic properties of pseudobinary alloys with $3 d$ elements is most useful for the understanding of the magnetism in these compounds. In particular it offers the possibility of studying the onset of magnetism as a function of alloy concentration. We will here report on theoretical results for the listed pseudobinary compounds. Comparison will be made with experimental results for the lattice constants, the magnetic moments and the critical concentration for the onset of magnetism. In Sec. II we describe the details of the calculations, Sec. III contains the results and the conclusions will be given in Sec. IV.

\section{DETAILS OF THE CALCULATIONS}

All the studied systems crystallize in the cubic Lavesphase ( $C 15$ structure) except for $\mathrm{UNi}_{2}$ which forms in the hexagonal Laves phase ( $C 14$ structure). The present self-consistent electronic-structure calculations were performed only for the $C 15$ crystal structure, which means that our treatment of $\mathrm{UNi}_{2}$ is only approximate. Except when explicitly stated the calculations were done for the experimental lattice constant for each of the pseudobinary systems. The energy bands were calculated using the linear muffin-tin orbitals (LMTO) technique and the calculations also included the combined correction terms to the atomic-sphere approximation (ASA). ${ }^{11}$ Final convergence was obtained using $89 \mathbf{k}$ points in an irreducible wedge of the Brillouin zone, and the density of states (DOS) was calculated using the analytical tetrahedron method $^{12}$ with 801 energy steps over a $1.0-\mathrm{Ry}$ broad energy window. The self-consistent potential was calculated with a frozen-core and the local-spin-density approximation was used for the exchange and correlation potential with the von Barth-Hedin parametrization. ${ }^{13}$ Relativistic effects were included in terms of mass velocity and Darwin shift, but the spin-orbit coupling was neglected.

The calculations for the disordered systems were done by simulating their electronic structure by that of an ordered compound with the same stoichiometry. ${ }^{14}$ The Wigner-Seitz cell of the yttrium (uranium) Laves phases contains 2 yttrium (uranium) atoms and 4 transitionmetal atoms. Considering, for instance, $\mathrm{Y}_{2} \mathrm{Fe}_{4}$ we can by progressive replacement of iron with cobalt study the magnetism in the $\mathrm{Y}\left(\mathrm{Fe}_{1-x} \mathrm{Co}_{x}\right)_{2}$ alloy system for $x=0$, $0.25,0.50,0.75$, and 1.0 .

\section{RESULTS}

\section{A. $\mathbf{Y}\left(\mathrm{Fe}_{1-x} \mathrm{Co}_{x}\right)_{2}$}

For the $\mathrm{Y}\left(\mathrm{Fe}_{1-x} \mathrm{Co}_{x}\right)_{2}$ system a preliminary report on some of our results has already been published. ${ }^{15}$ Here we will give a more complete account. This pseudobinary alloy is of a particular interest to study since it can be used as a reference for the calculations of the corresponding uranium systems, which are considerably more complex due to the presence of the $5 f$ electrons.

The calculated magnetic moments for the ordered compounds $\mathrm{Y}_{2} \mathrm{Fe}_{4}, \mathrm{Y}_{2} \mathrm{Fe}_{3} \mathrm{Co}, \mathrm{Y}_{2} \mathrm{Fe}_{2} \mathrm{Co}_{2}, \mathrm{Y}_{2} \mathrm{FeCo}_{3}$, and $\mathrm{Y}_{2} \mathrm{Co}_{4}$ are shown in Fig. 1 together with experimental data for the pseudobinary alloys. 4,16 The calculated total moment for $\mathrm{YFe}_{2}$ is $2.94 \mu_{B}$, which is in good agreement

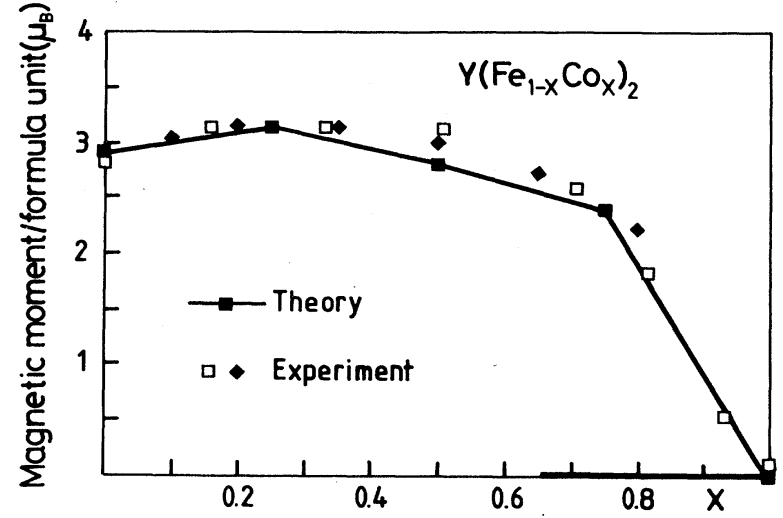

FIG. 1. Total magnetic moment for $\mathrm{Y}\left(\mathrm{Fe}_{1-x} \mathrm{Co}_{x}\right)_{2}$. Theoretical values are denoted by filled squares and experimental values by open squares (Piercy et al., Ref. 16) or filled tilted squares (Steiner et al., Ref. 16). The fat part of the $x$ axis is the concentration region where the paramagnetic state is locally stable.

with the measured value of $2.91 \mu_{B} \cdot{ }^{7}$ The trend of the measured total magnetic moment as a function of composition, with a maximum at approximately $x=0.30$, is also reproduced. From Table $I$ it becomes clear that to a large extent this maximum is connected with the $\mathrm{Fe}$ moment, whereas the $\mathrm{Y}$ and Co moments stay more or less constant at $-0.4 \mu_{B}$ and $1.2 \mu_{B}$, respectively, as long as there is magnetic order in the system. The negative moment on the yttrium atom is due to different hybridization between the $3 d$ and the $4 d$ orbitals for the majority and the minority spin bands. ${ }^{16}$

The paramagnetic state densities (DOS) for the studied compounds are found to be very similar to each other, with a band predominantly composed of $3 d$ orbitals lying below a broader band dominated by yttrium $4 d$ orbitals (Fig. 2). The separation between these two features is not distinct since the hybridization between the $3 d$ and $4 d$ states is strong. As we proceed from one compound to the next, substituting iron with cobalt, we add valence electrons and a progressive filling of the $3 d$-dominated

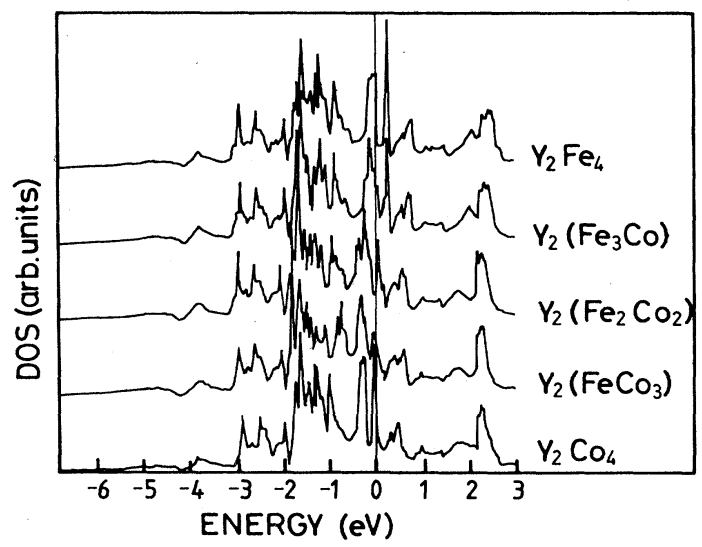

FIG. 2. Total DOS for the ordered compounds $\mathrm{Y}_{2} \mathrm{Fe}_{4}$, $\mathrm{Y}_{2} \mathrm{Fe}_{3} \mathrm{Co}, \mathrm{Y}_{2} \mathrm{Fe}_{2} \mathrm{Co}_{2}, \mathrm{Y}_{2} \mathrm{FeCo}_{3}$, and $\mathrm{Y}_{2} \mathrm{Co}_{4}$ in the paramagnetic state (for details see text). Energies are in $\mathrm{eV}$ and the Fermi level is at zero energy. 
TABLE I. Calculated total and individual ( $\mathrm{Y}, \mathrm{Fe}$, and $\mathrm{Co}$ ) magnetic moments, spin-projected $3 d$ occupation numbers, total $3 d$ occupation number per $3 d$ atom, $3 d$ partial pressure, and loss of bonding due to the magnetic moments for the ordered compounds $\mathrm{Y}_{2} \mathrm{Fe}_{4}, \mathrm{Y}_{2} \mathrm{Fe}_{3} \mathrm{Co}, \mathrm{Y}_{2} \mathrm{Fe}_{2} \mathrm{Co}_{2}, \mathrm{Y}_{2} \mathrm{FeCo}_{3}$, and $\mathrm{Y}_{2} \mathrm{Co}_{4}$.

\begin{tabular}{lccccr}
\hline & $\mathrm{YFe}_{2}$ & $\mathrm{YFe}_{3 / 2} \mathrm{Co}_{1 / 2}$ & $\mathrm{YFeCo}$ & $\mathrm{YFe}_{1 / 2} \mathrm{Co}_{3 / 2}$ & $\mathrm{YCo}$ \\
\hline$\mu_{\text {tot }}\left(\mu_{B} / Y\right.$ atom $)$ & 2.94 & 3.22 & 2.82 & 2.39 & \\
$\mu(\mathrm{Fe})\left(\mu_{B}\right)$ & 1.68 & 2.04 & 2.01 & 1.91 & \\
$\mu(\mathrm{Co})\left(\mu_{B}\right)$ & & 1.22 & 1.23 & 1.21 & \\
$\mu(\mathrm{Y})\left(\mu_{B}\right)$ & -0.43 & -0.45 & -0.42 & -0.38 & \\
$n_{3 d}($ majority $)$ & 8.4 & 8.8 & 8.8 & 8.8 & \\
$n_{3 d}($ minority) & 4.9 & 5.0 & 5.5 & 5.9 & 7.37 \\
$n_{3 d} / 3 d$ atom & 6.64 & 6.87 & 7.13 & -0.56 & -0.50 \\
$\left(P_{d} V\right)_{\text {para }}(\mathrm{Ry})$ & -0.67 & -0.63 & -0.59 & 18 & 0 \\
$(P)_{\text {magn }}$ (kbar) & 40 & 48 & 36 & & \\
\hline
\end{tabular}

band takes place. The similarity of the state densities suggests that a rigid band model is a good approximation. According to our calculations the low lying occupied $d$ band in all these compounds holds-per $\mathrm{Y}\left(\mathrm{Fe}_{1-x} \mathrm{Co}_{x}\right)_{2}$ formula unit-approximately $1.6 \mathrm{Y} 4 d$ electrons. We also find a $3 d$ occupation number $n_{3 d}$ approximately obeying the following linear relation:

$$
n_{3 d}=2[6.64(1-x)+7.74 x]
$$

Here 6.64 and 7.74 are the $d$ occupations per atom for $\mathrm{Fe}$ and Co, respectively.

To understand the origin of the magnetization curve (Fig. 1) we now construct a model DOS for these systems which for simplicity contains only the $d$ states (Fig. 3). The width and position for this model DOS are obtained from the bottom, center, and top band parameters from our paramagnetic self-consistent band calculation for $\mathrm{YFe}_{2}$ (obtained by using the Wigner-Seitz rule). ${ }^{11}$ Hence this model DOS consists of two hybridizing and slightly separated bands (rectangles), where the lower band mainly originates from $3 d$ orbitals and the upper from $4 d$ orbitals. The lower rectangle of width $0.24 \mathrm{Ry}$ can hold a

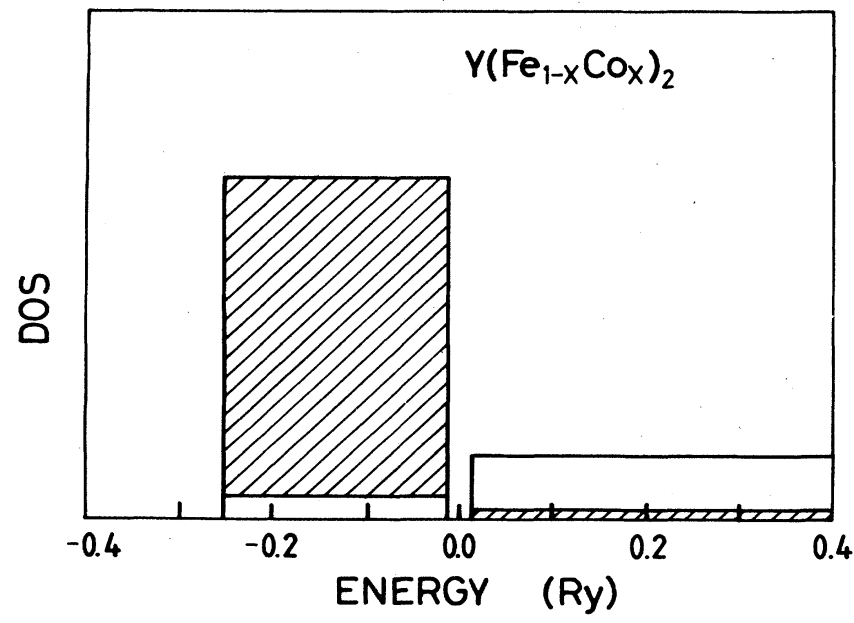

FIG. 3. Model DOS for the $\mathrm{Y}\left(\mathrm{Fe}_{1-x} \mathrm{Co}_{x}\right)_{2}$ alloys in the paramagnetic state (for details see text). The shaded region shows the $3 d$ partial DOS. Energies are in Ry. total of 20 electrons, of which approximately 1.6 are yttrium $d$ electrons. Since the bandwidth decreases with atomic number, one would expect the $3 d$ moment to increase with $x$ in $\mathrm{Y}\left(\mathrm{Fe}_{1-x} \mathrm{Co}_{x}\right)_{2}$, until the majority spin band becomes filled. This will correspond to a maximum in the magnetic moment and will be followed by a decrease of the moment with $x$, since now the minority spin band has to be filled (Slater-Pauling curve). This means that when we arrive at the point where the number of $3 d$ electrons is equal to $(20-1.6) / 2=9.2$ in the majority spin band there is a maximum in the $3 d$ spin moment. From a calculated exchange splitting of $0.11 \mathrm{Ry}$, we find by means of simple algebra [using Eq. (1)] that for the schematic DOS model this maximum corresponds to an $x$ value of 0.4 , which is in fair agreement with experiment.

An analogy with a Slater-Pauling curve concerning the magnetism of these systems was also discussed in Ref. 17. Considering the individual moments we find that the moment on Co actually corresponds to an almost saturated spin situation. Its majority band occupation is 4.5 which is close to the highest possible value which is $9.2 / 2=4.6$. Since the calculated moment on $\mathrm{Fe}$ is not quite $1 \mu_{B}$ higher than for $\mathrm{Co}$, this means that its moment is not quite fully saturated at any alloy concentration. Therefore when alloying the magnetic compound $\mathrm{YFe}_{2}$ with the nonmagnetic $\mathrm{YCo}_{2}$ compound, there is an induced magnetism for $\mathrm{YCo}_{2}$ which is even closer to magnetic saturation than $\mathrm{YFe}_{2}$ itself, despite the fact that it is the latter compound which forces $\mathrm{YCo}_{2}$ to become magnetic. This strongly suggests that there is a metamagnetic state in $\mathrm{YCo}_{2}$ with a filled majority band. It is also interesting to notice that there is a boot-strapping effect in the magnetic alloys, in the sense that the induced magnetism for $\mathrm{YCo}_{2}$ enhances the magnetism on $\mathrm{YFe}_{2}$ (Table I).

Now we turn to the ferromagnetic-paramagnetic transition as a function of concentration in $\mathrm{Y}\left(\mathrm{Fe}_{1-x} \mathrm{Co}_{x}\right)_{2}$. This is first investigated by calculating the Stoner product $^{18}$ for the paramagnetic state of the various ordered ternary compounds and then interpolating between these values to give a Stoner product for intermediate concentrations (Fig. 4). A critical concentration for the disappearance of magnetism is found to be $65 \%$ Co substitution. This should be compared to the experimental values of about $95 \%$ (Ref. 17). This large discrepancy 


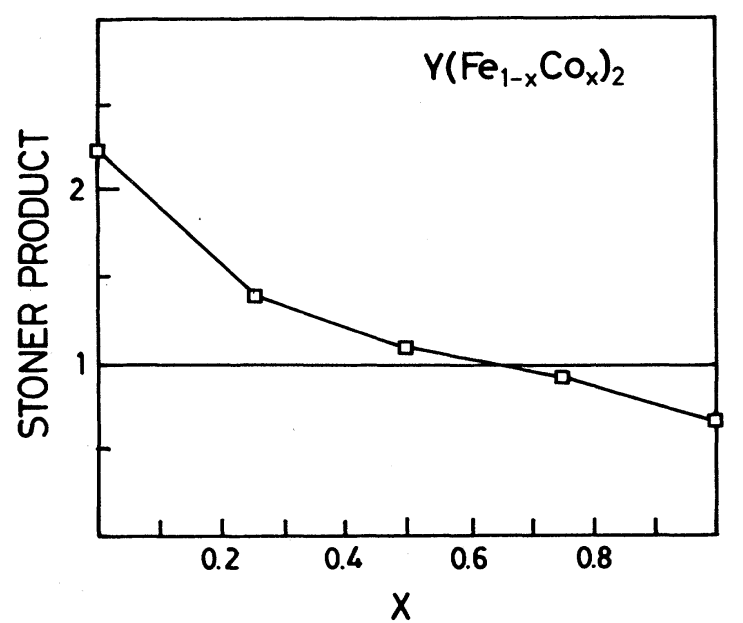

FIG. 4. Calculated Stoner products for the ordered compounds $\mathrm{Y}_{2} \mathrm{Fe}_{4}, \mathrm{Y}_{2} \mathrm{Fe}_{3} \mathrm{Co}, \mathrm{Y}_{2} \mathrm{Fe}_{2} \mathrm{Co}_{2}, \mathrm{Y}_{2} \mathrm{FeCo}_{3}$, and $\mathrm{Y}_{2} \mathrm{Co}_{4}$. The solid line represents the interpolated values.

suggests that the magnetic states in these heavily Co substituted compounds are similar to those in $\mathrm{YCo}_{2},{ }^{5}$ namely that there are two competing locally stable states, the paramagnetic and the ferromagnetic one. This must be a first-order transition as a function of alloy concentration. To investigate this possibility we have performed fixed-spin-moment (FSM) ${ }^{19}$ calculations for $\mathrm{Y}_{2} \mathrm{FeCo}_{3}$, by computing the total energy as a function of magnetic moment. The results of these calculations are shown in Fig. 5 from which it is clear that there are indeed two local energy minima. The high-spin state has the lowest energy although the energy barrier between the two states is rather small. From this we conclude that the magneticto-nonmagnetic transition takes place somewhere in the range $0.75<x<1.0$ in agreement with magnetic data. ${ }^{17}$ Interpolation of the energy difference separating the high- and low-spin states between $\mathrm{YCo}_{2}$ (calculated by

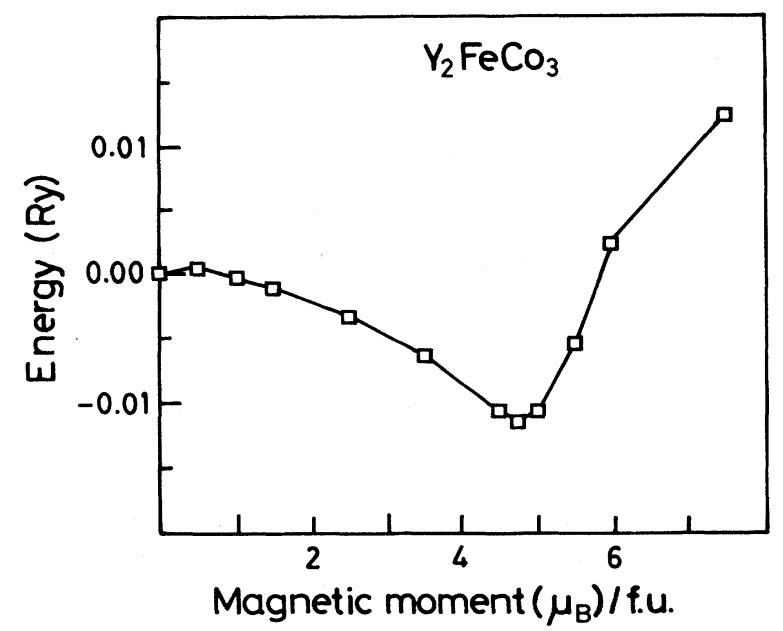

FIG. 5. Calculated (FSM) energy of $\mathrm{Y}_{2} \mathrm{FeCo}_{3}$ as a function of total magnetic moment. The positive curvature of the energy curve at zero moment is consistent with our calculated Stoner product being less than one.
Schwarz and Mohn using the FSM technique ${ }^{5}$ ) and $\mathrm{Y}_{2} \mathrm{FeCo}_{3}$ yields a critical concentration of 0.97 , which is very close to the experimental value.

In Fig. 6 we plot the experimental lattice constant as a function of $x$ in the $\mathrm{Y}\left(\mathrm{Fe}_{1-x} \mathrm{Co}_{x}\right)_{2}$ system, and an anomalous nonlinear behavior is noted. We ascribe this to magnetovolume effects ${ }^{20}$ and to quantify this conjecture we calculate the decrease in bonding $d$ pressure $\left(P_{d}<0\right)$ due to a band magnetism $m_{3 d}$ [per $\mathrm{Y}\left(\boldsymbol{M}_{1-x} \boldsymbol{M}_{x}^{\prime}\right)_{2}$ formula unit cell] of the $3 d$ electrons; ${ }^{21}$

$$
\begin{aligned}
& \left(P_{d} V\right)_{\text {magn }}=\left[m_{3 d}^{2} / 20 n_{3 d}\left(1-n_{3 d} / 20\right)\right]\left(-P_{d} V\right)_{\text {para }}, \\
& m_{3 d}=n_{3 d} \text { (majority) }-n_{3 d} \text { (minority), }
\end{aligned}
$$

where $n_{3 d}$ (majority) [ $n_{3 d}$ (minority)] is the number of majority (minority) $3 d$ electrons (Table $\mathrm{I}$ ) and $n_{3 d}$ is given by Eq. (1).

From a paramagnetic calculation we compute the $3 d$ pressure, i.e., the sum of the $\mathrm{Fe} 3 d$ and Co $3 d$ pressures, and this is tabulated as $\left(P_{d} V\right)_{\text {para }}$ in Table I. From our equation of state calculations we obtained a magnetovolume effect of $1.75 \AA^{3}$ in $\mathrm{YFe}_{2}$ (corresponding to a $3.5 \%$ volume expansion) and zero for $\mathrm{YCo}_{2}\left(\mathrm{YCo}_{2}\right.$ being nonmagnetic). (This theoretical value for $\mathrm{YFe}_{2}$ agrees well with an estimated magnetovolume effect of about 1.5-2 $\AA^{3}{ }^{22}$ ) Thus if these pseudobinary alloys were all nonmagnetic, and assuming the validity of Vegard's law ${ }^{23}$ for the hypothetical paramagnetic state, the volumes would follow the line labeled "Vegard" in Fig. 6. In Fig. 7 we plot the difference between the experimental volume and the "Vegard" volume, and this difference defines the quasiexperimental magnetovolume effect. Using Eq. (2) we now calculate the theoretical increase in $3 d$ pressure $\left(P_{d}\right)_{\text {magn }}$ caused by the magnetism (Table I). For $\mathrm{YFe}_{2}$ we calculate $d P / d V$ to be $24 \mathrm{kbar} / \AA^{3}$, and assuming that this value is approximately the same for all the studied alloys, we can now calculate the increase in volume due to magnetism as $\left(P_{d}\right)_{\text {magn }} / 24\left(\AA^{3}\right)$. These theoretical volumes are compared with experiments in Fig. 7 and the agreement is good. It is also clear from this graph that

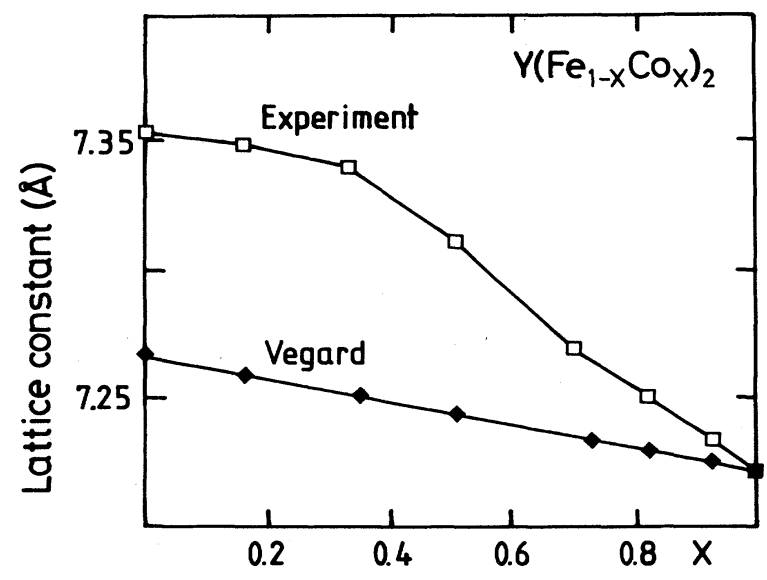

FIG. 6. Experimental and estimated (Vegard's law, for details see text) lattice constants for $\mathrm{Y}\left(\mathrm{Fe}_{1-x} \mathrm{Co}_{x}\right)_{2}$. 


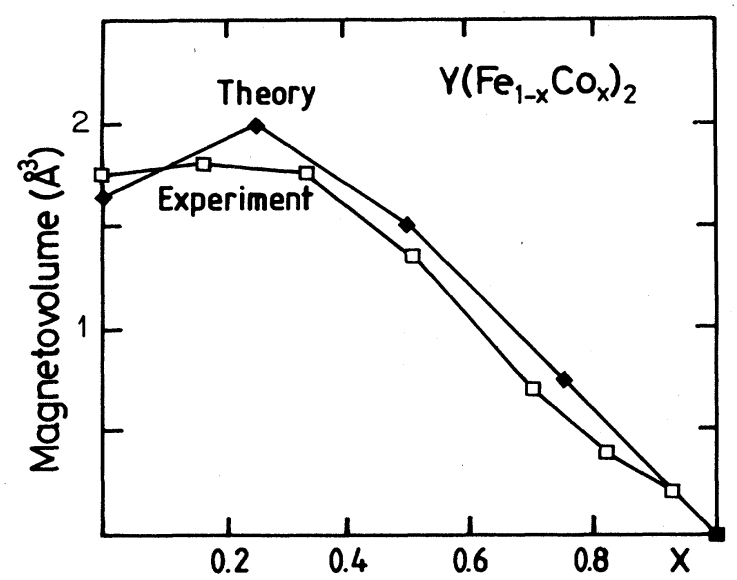

FIG. 7. Theoretical and quasiexperimental magnetovolumes for $\mathrm{Y}\left(\mathrm{Fe}_{1-x} \mathrm{Co}_{x}\right)_{2}$ (for details see text).

the largest experimental deviation from Vegard's law occurs for a concentration $x \approx 0.30$, which simultaneously gives the largest magnetic moment. A similar behavior of the volumes in the pseudobinary alloy system $\operatorname{Er}\left(\mathrm{Fe}_{1-x} \mathrm{Co}_{x}\right)_{2}$ has also been observed, ${ }^{24}$ and we suggest the same mechanism to be responsible for this effect.

The procedure we have used for calculating the electronic structure of the present pseudobinary systems neglects the effect of disorder. In order to investigate some aspects of the validity of this approximation we have looked to other approximate methods for calculating the electronic structure of alloys. The simplest way this can be done is to replace the true system by an average, where the site potential is replaced by an average potential. ${ }^{25}$ We have, therefore, used the virtual-crystal approximation (VCA) for the $\mathrm{Y}\left(\mathrm{Fe}_{0.75} \mathrm{Co}_{0.25}\right)_{2}$ system and compared its DOS with the DOS of the present cluster calculation. Thus we calculate the electronic structure for the $\mathrm{YM}_{2}$ compound where the atomic number $Z$ of metal $M$ is equal to 26.25 . The results are shown in Fig. 8 , and we find that the two methods compare well, even concerning the finer details of the electronic structure.

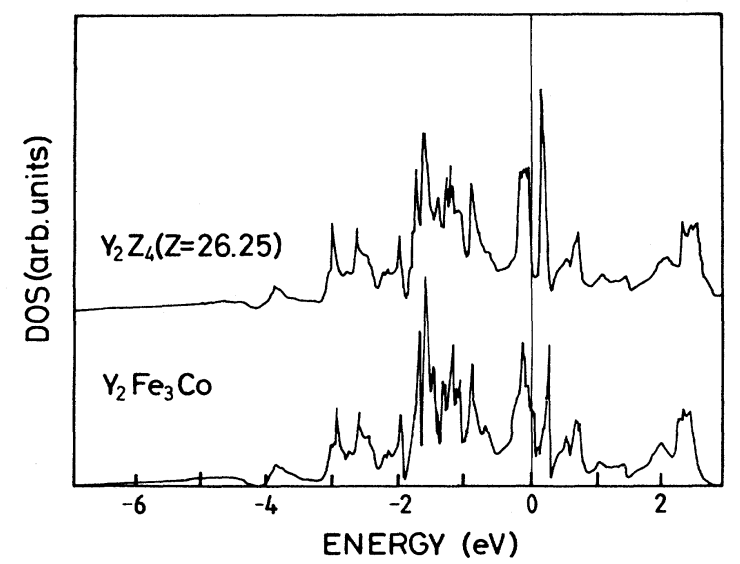

FIG. 8. Total DOS for the ordered compound $\mathrm{Y}_{2} \mathrm{Fe}_{3} \mathrm{Co}$ and $\mathrm{Y}_{2} \mathrm{Z}_{4}$ where $Z$ is a fictitious atom type with nuclear charge $z=26.25$. Energies are in $\mathrm{eV}$ and zero is at the Fermi level.

$$
\text { B. } \mathrm{U}\left(\mathrm{Fe}_{0.5} \mathrm{Ni}_{0.5}\right)_{2}
$$

The good results for the $\mathrm{Y}\left(\mathrm{Fe}_{1-x} \mathrm{Co}_{x}\right)_{2}$ alloy system encouraged us to proceed to the more complex systems involving uranium. The first pseudobinary system we will discuss is the paramagnetic $\mathrm{U}\left(\mathrm{Fe}_{0.5} \mathrm{Ni}_{0.5}\right)_{2}$ system. This system crystallizes both in a cubic and hexagonal Laves phase, with almost identical volumes, ${ }^{1}$ but we will only consider the cubic phase. A slight complication is also the fact that $\mathrm{UNi}_{2}$ crystallizes in the $C 14$ structure. However for the present purpose where we consider integrated properties like pressure and magnetic moment, the fact that the calculations are done for the incorrect $C 15$ phase is not expected to introduce any appreciable error. In Fig. 9 we plot both the experimental and theoretical volumes for $\mathrm{UFe}_{2}, \mathrm{UFeNi}, \mathrm{UNi}_{2}$, and $\mathrm{UCo}_{2}$. The reason we include $\mathrm{UCo}_{2}$ is that this compound is the virtual-crystal approximation (VCA) to the pseudobinary $\mathrm{U}\left(\mathrm{Fe}_{0.5} \mathrm{Ni}_{0.5}\right)_{2}$ system. In Fig. 9 we also show the volume Vegard's law would give with $\mathrm{UFe}_{2}$ and $\mathrm{UNi}_{2}$ as end systems (theoretically and experimentally). Here we see that the experimental volume of $\mathrm{U}\left(\mathrm{Fe}_{0.5} \mathrm{Ni}_{0.5}\right)_{2}$ is in rather good agreement with Vegard's law. ${ }^{26}$ In this connection we also note that the theoretical volume for $\mathrm{UFeNi}$ agrees well with Vegard's law applied to the theoretical values for the end systems in their paramagnetic states. The VCA for $\mathrm{U}\left(\mathrm{Fe}_{0.5} \mathrm{Ni}_{0.5}\right)_{2}$ is as mentioned $\mathrm{UCo}_{2}$ and the theoretical as well as experimental volume for this VCA compound is far from the experimental one for $\mathrm{U}\left(\mathrm{Fe}_{0.5} \mathrm{Ni}_{0.5}\right)_{2}$. It is especially worth noticing the deviation between the VCA volume and the volume for the or-

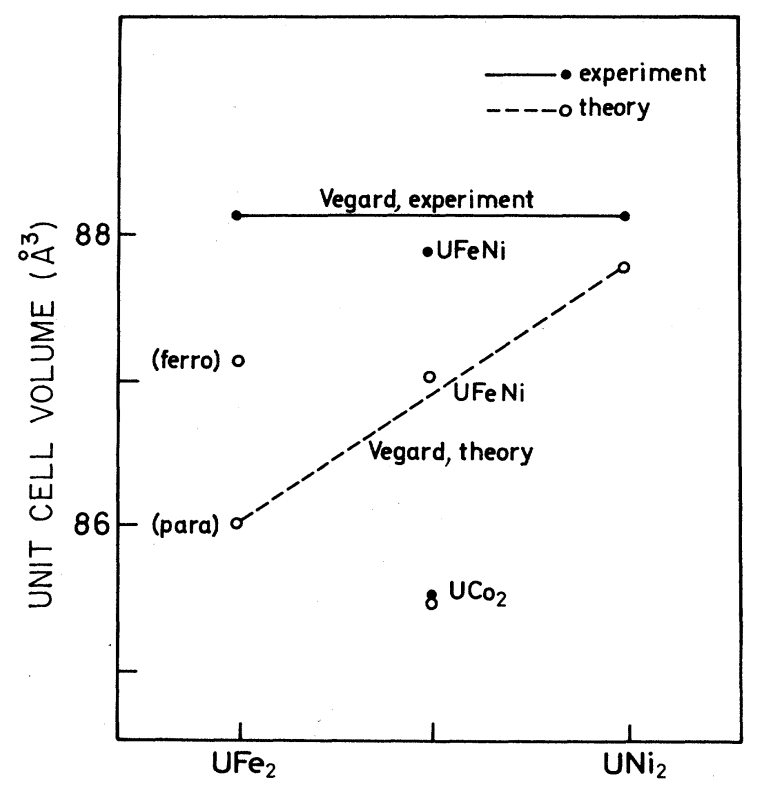

FIG. 9. Solid black curve represents the estimated volumes for the $\mathrm{U}\left(\mathrm{Fe}_{1-x} \mathrm{Ni}_{x}\right)_{2}$ alloys (Vegard's law, for details see text). Also given are the experimental volumes for $\mathrm{UFe}_{2}, \mathrm{UFeNi}$, $\mathrm{UCo}_{2}$, and $\mathrm{UNi}_{2}$ (black dots). Theoretical volumes for the ordered systems are indicated with open circles. The estimated paramagnetic volumes (Vegard's law) for $\mathrm{U}\left(\mathrm{Fe}_{1-x} \mathrm{Ni}_{x}\right)_{2}$, based upon the theoretical volumes of $\mathrm{UFe}_{2}$ and $\mathrm{UNi}_{2}$, are indicated with a broken line. 
dered ternary compound. In order to elucidate the reason for this difference we plot schematically in Fig. 10 the partial $3 d$ DOS for $\mathrm{Fe}$ and $\mathrm{Ni}$ in $\mathrm{UFeNi}$ and the $3 d$ DOS for $\mathrm{Co}$ in $\mathrm{UCo}_{2}$. The band positions and the band widths for all these systems were obtained from our selfconsistent calculations, using the approximate WignerSeitz rule. ${ }^{11}$ Figure 10 shows that the $3 d$ partial bands in $\mathrm{UFeNi}$ and $\mathrm{UCO}_{2}$ can be described as low-lying bonding states and the higher-lying $d$ bands (not shown), mainly originating from the $\mathrm{U} 6 d$ states, as antibonding. In addition to the $d$ states there are, of course, also $s$ and $p$ states. However, for our arguments these states play a minor role, and we will disregard them. It is more difficult to argue that the $f$ states also can be neglected, and it is only from a detailed inspection of the $f$ contribution to the bonding can it be seen that for the present discussion they play only a secondary role. From Fig. 10 it is clear that the maximum bonding situation occurs in $\mathrm{UCO}_{2}$. This is so since here almost all the bonding orbitals are filled and additional valence electrons would have to enter antibonding states. Consequently $\mathrm{UCo}_{2}$ has the smallest volume of the uranium $3 d$ Laves phases. Replacing the bonding Co $3 d$ band by superposed $\mathrm{Fe}$ and $\mathrm{Ni}$ $3 d$ bands, i.e., using a cluster approach instead of the VCA, shows that the total binding is reduced. In order to quantify this we consider the electronic pressure $P_{l}$ from the $l$ th orbital using the simple first-order pressure relation: ${ }^{21}$

$3 P_{l} V=n_{l}\left(-\frac{\partial C_{l}}{\partial \ln S}\right)+n_{l}\left(\bar{E}_{l}-C_{l}\right)\left(-\frac{\partial \ln w_{l}}{\partial \ln S}\right)$

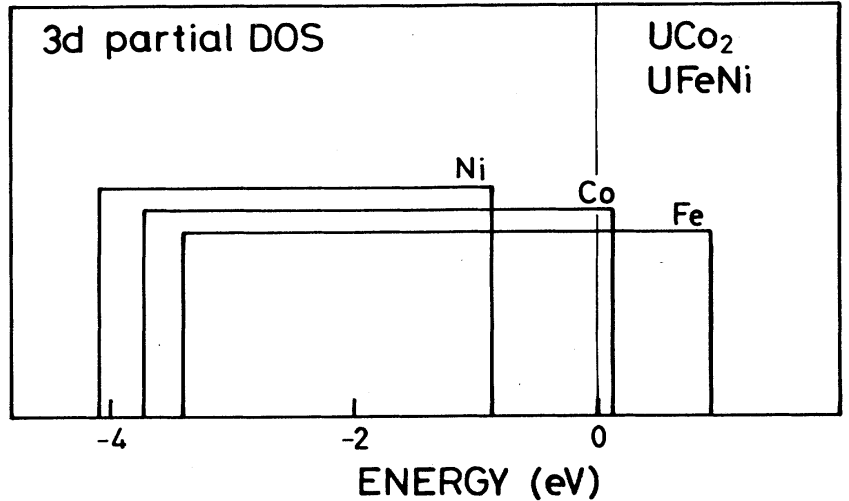

FIG. 10. The bonding part of the model $3 d$ partial DOS for $\mathrm{UCO}_{2}$ and $\mathrm{UFeNi}$ (for details see text). Energies are given in $\mathrm{eV}$ and zero is at the Fermi level.

Here $n_{l}$ is the number of electrons in the $l$ orbital, $C_{l}$ is the center of the $l$ band, $w_{l}$ is the band width, $S$ is the Wigner-Seitz radius, and $\bar{E}_{l}$ is the center of the occupied part of the $l$ band. The values of the different terms in Eq. (3) are given in Table II for the $3 d$ states. Here we see that, mainly due to the second term in Eq. (3), the bonding term is reduced in $\mathrm{UFeNi}$ as compared to $\mathrm{UCo}_{2}$. The difference is $0.07 \mathrm{Ry}$, which corresponds to $18 \mathrm{kbar}$. Using the full pressure formula we calculate the same difference to be $20 \mathrm{kbar}$. Hence we find that UFeNi has less bonding properties as compared to its virtual crystal, $\mathrm{UCo}_{2}$, and therefore has a larger volume. The reason Eq.

TABLE II. The upper part gives the calculated partial $3 d$ pressure $\left(P_{3 d} V\right)_{\text {tot }}$ for $\mathrm{UCO}_{2}$ and $\mathrm{UFeNi}$ using the full-pressure formula, and calculated difference in bonding $3 d$ pressure $\triangle P\left(\mathrm{UCO}_{2}-\mathrm{UFeNi}\right.$ ) (for details see text). The lower part gives the $3 d$ partial pressure from the first-order pressure relation [Eq. (3)] together with the contribution from the band center $\left(P_{3 d} V\right)_{c}$ and the band width $\left(P_{3 d} V\right)_{w}$. $\left(\bar{E}_{3 d}-C_{3 d}\right)$ is the difference between the center of the occupied part of the $3 d$ band and the center of the $3 d$ band. $n_{3 d}$ is the $3 d$ occupation number and $w_{3 d}$ is the $3 d$ bandwidth. The computed difference in the bonding $3 d$ pressure is denoted $\Delta P\left(\mathrm{UCo}_{2}-\mathrm{UFeNi}\right)_{0}$. When appropriate, the sum of the contributions from $\mathrm{Fe}$ and $\mathrm{Ni}$ in $\mathrm{UFeNi}$ is also listed.

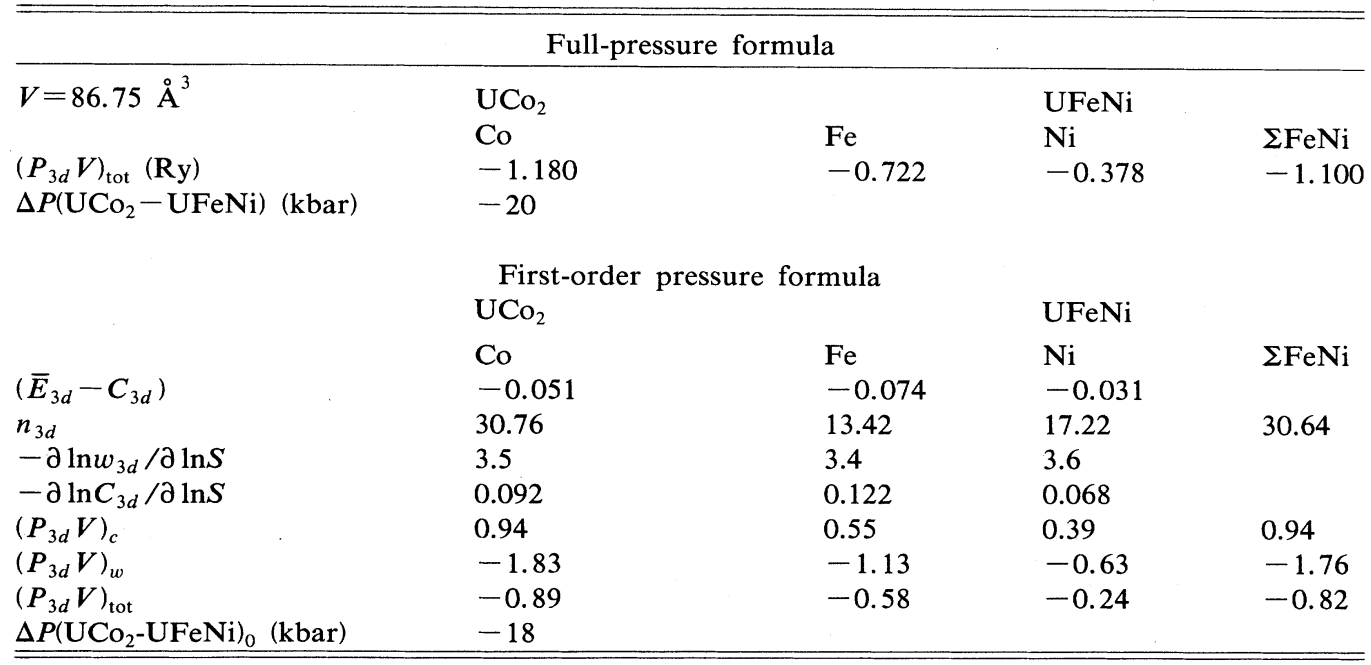


(3) gives a larger negative value for $\mathrm{UCo}_{2}$ as compared to UFeNi can be simply seen as follows. Since the second term in Eq. (3) depends parabolically on the $d$ occupation number, it follows that replacing the average pressure with the pressure at the average occupation number always increases the bonding.

In order to further compare the electronic structure of $\mathrm{UFeNi}$ with its virtual crystal $\mathrm{UCo}_{2}$, we show in Fig. 11 their calculated DOS. As can be seen both systems are dominated by the $3 d$ band at energies varying from -4 to $+2 \mathrm{eV}$ and by a relatively broad $5 f$ band which is pinned at the Fermi energy $E_{F}$ but has its main weight between 0 and $1.5 \mathrm{eV}$. However $\mathrm{UCo}_{2}$ shows some pronounced structures at energies around $-1 \mathrm{eV}$, which are absent or smeared out in UFeNi. Furthermore the structure at $-3 \mathrm{eV}$ in $\mathrm{UFeNi}$ is located at slightly less binding energy in $\mathrm{UCO}_{2}$. Thus there are differences in the details between the state densities of these two approximations, but the overall shapes are very similar.

The magnetic behavior of the $\mathrm{U}\left(\mathrm{Fe}_{0.5} \mathrm{Ni}_{0.5}\right)_{2}$ alloy is most interesting. Although the alloy is composed of two constituents both of which are ferromagnetic it is a paramagnet. In the discussion above about Vegard's law we neglected the fact that $\mathrm{UNi}_{2}$ is a ferromagnet. However this could be done without an appreciable error since this effect is here small, simply because the moment is very small. Actually our calculations for $\mathrm{UNi}_{2}$ give a ferromagnetic state which originates from a small spin polarization of the $5 f$ band. Thus the ferromagnetism in $\mathrm{UFe}_{2}$ and $\mathrm{UNi}_{2}$ are of very different origin, since in $\mathrm{UFe}_{2}$ the magnetism is driven by the $\mathrm{Fe} 3 d$ electrons. It is this difference in the origin of the magnetism which explains the disappearance of the magnetism in the $x=0.5$ alloy. The fact that the Ni $3 d$-based band is filled in $\mathrm{UNi}_{2}$ decreases the overlap energy between the $5 f$ and $3 d$ states. This causes a reduction of the $5 f-3 d$ hybridization and it

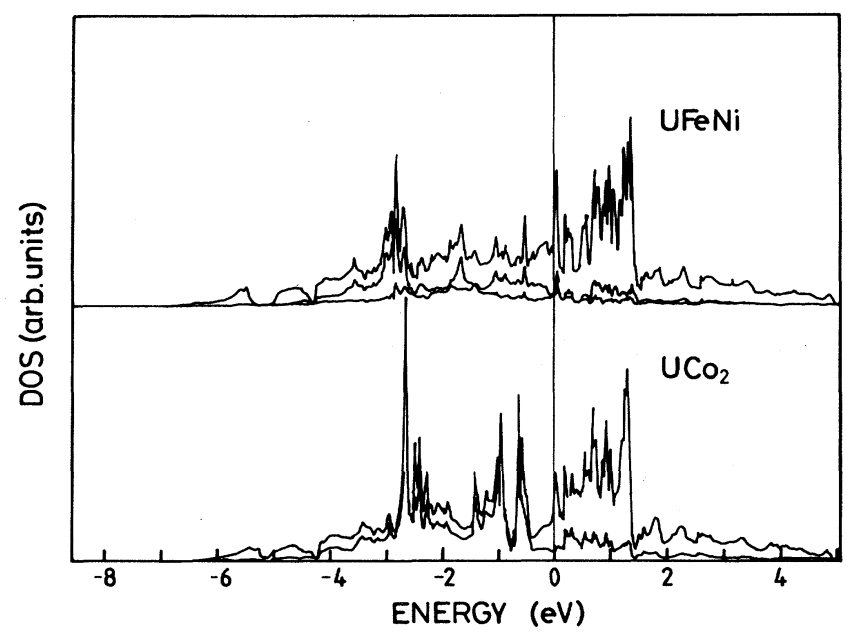

FIG. 11. Total (upper curve in each panel) and $3 d$ partial (lower curve in each panel) DOS for $\mathrm{UCo}_{2}$ and $\mathrm{UFeNi}$. The $3 d$ partial DOS for UFeNi is decomposed into the $\mathrm{Ni}$ and Fe components. The $\mathrm{Ni}$ band has its major weight at energies about 3 $\mathrm{eV}$ below the Fermi level, whereas the $\mathrm{Fe}$ band is more widespread. Energies are in $\mathrm{eV}$ and zero is at the Fermi level. is actually this narrowing of the $f$ band which gives rise to the ferromagnetism in $\mathrm{UNi}_{2}$. By substituting $\mathrm{Ni}$ with Fe the $5 f-3 d$ hybridization increases and the $5 f$ spin moment disappears. In the other end of the pseudobinary alloy the $5 f$ states oppose the $3 d$ spin polarization and cause a reduction of the iron magnetic moment in $\mathrm{UFe}_{2}$ as compared to $\mathrm{YFe}_{2}$. When $\mathrm{Fe}$ is substituted with $\mathrm{Co}$ the extra valence electrons enter the $3 d$ bonding band. As we have already shown above, for the $\mathrm{Y}\left(\mathrm{Fe}_{1-x} \mathrm{Co}_{x}\right)_{2}$ system this substitution eventually leads to the disappearance of the magnetism. Since for $\mathrm{UFe}_{2}$ the magnitude of the Fe moment is already quite low, the Co substitution is expected to destroy the magnetism more rapidly with $x$ in $\mathrm{U}\left(\mathrm{Fe}_{1-x} \mathrm{Co}_{x}\right)_{2}$ than in $\mathrm{Y}\left(\mathrm{Fe}_{1-x} \mathrm{Co}_{x}\right)_{2}$. This is also observed experimentally. ${ }^{1}$ Since $\mathrm{Ni}$ has one more valence electron than $\mathrm{Co}, \mathrm{Ni}$ substitution would be even more effective in reducing the moment. This is confirmed by our calculations for UFeNi where the Stoner product is found to be less than one $(0.96)$ and from this the paramagnetic behavior can be understood. However since both $\mathrm{UFe}_{2}$ and $\mathrm{UNi}_{2}$ are ferromagnets one might suspect that magnetic ordering in UFeNi at least can be realized when an external magnetic field is applied (metamagnetism).

To investigate this possibility we have made a fixed-spin-moment (FSM) calculation ${ }^{19}$ also for UFeNi. As already mentioned, in this treatment the magnetic moment $M$ is considered as a free variable and the total energy is calculated as a function of $M$. If local minima are found for nonzero values of $M$ these can always be reached by sufficiently strong applied fields. The results of our FSM calculation of UFeNi show a very flat minimum at $M=0$ in accordance with the enhanced paramagnetic ground state. However with increasing magnetic moments the energy increases monotonically, and thus no metamagnetic behavior was found from our calculations for UFeNi. Again this might be compared with $\mathrm{YNi}_{2}$ where we do not expect any metamagnetic behavior. Similarly, for $\mathrm{Y}\left(\mathrm{Co}_{1-x} \mathrm{Ni}_{x}\right)_{2}$ the metamagnetism predicted for $x=0$ will disappear with increasing $x$.

$$
\text { C. } \mathbf{U}\left(\mathrm{Fe}_{1-x} \mathrm{Mn}_{x}\right)_{2}
$$

For some of the Laves-phase compounds between uranium and $3 d$ elements quite complicated magnetic structures can be expected, since if the compound is magnetic both transition and actinide metals are likely to carry a magnetic moment. Forming ternary compounds of the kind we are discussing here further complicates the situation. An explanation of the origin of these magnetic properties constitutes a severe test of band theory. In order to calculate a critical concentration for the disappearance of magnetic moments in these systems we compute the multiband Stoner integral ${ }^{18}$ and multiply it with the total DOS at $E_{F}$. The so obtained values of the Stoner product for $\mathrm{U}\left(\mathrm{Fe}_{1-x} \mathrm{Mn}_{x}\right)_{2}$ are plotted in Fig. 12, and from interpolation we find that the Stoner product is larger than one only when $x<0.28$, i.e., the alloys should only be magnetic when $x<0.28$. This assumes, of course, that the onset of magnetism is a second-order transition as a function of the concentration $x$. The cal- 


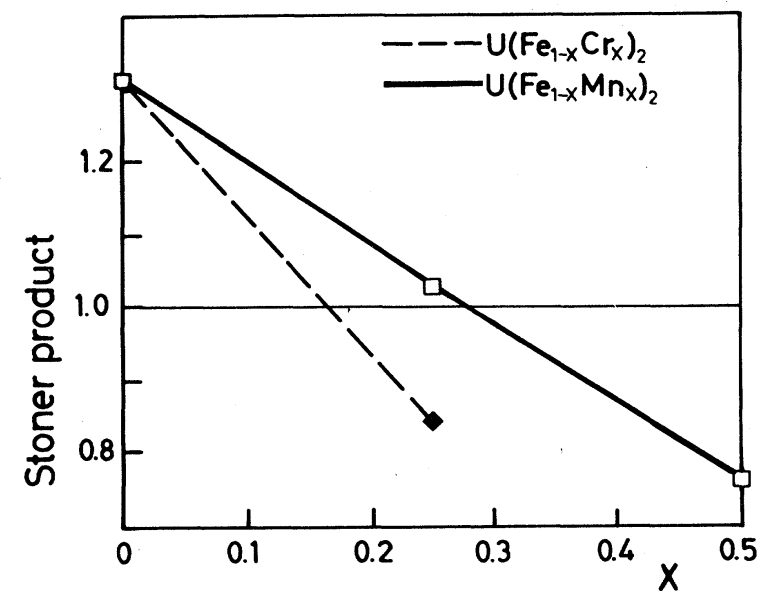

FIG. 12. Calculated Stoner product for the ordered systems $\mathrm{U}_{2} \mathrm{Fe}_{4}, \mathrm{U}_{2} \mathrm{Fe}_{3} \mathrm{Mn}, \mathrm{U}_{2} \mathrm{Fe}_{2} \mathrm{Mn}_{2}$ (open squares) and $\mathrm{U}_{2} \mathrm{Fe}{ }_{3} \mathrm{Cr}$ (solid tilted square). The solid and broken lines represent the interpolated values for the $\mathrm{Mn}$ and $\mathrm{Cr}$ substituted alloys, respectively.

culated value should be compared with an experimental critical concentration of $x=0.3$. $^{1,4}$ It is also worth noticing that for the pure $\mathrm{UFe}_{2}$ compounds we calculate the uranium spin moment to be $-0.6 \mu_{B}$ and the iron moment to be $0.8 \mu_{B}$, i.e., the uranium moment is antiparallel to the iron moment. However, for a proper treatment of the absolute magnitude of the uranium magnetic moment it is necessary to include the orbital contribution which is induced through the spin-orbit coupling. This has been fully elucidated elsewhere. ${ }^{27}$

From Fig. 13 it becomes apparent that a rigid-band model fairly well describes the electronic structure of the pseudobinary alloy system $\mathrm{U}\left(\mathrm{Fe}_{1-x} \mathrm{Mn}_{x}\right)_{2}$ since the plotted paramagnetic DOS for $\mathrm{UFe}_{2}, \mathrm{U}_{2} \mathrm{Fe}_{3} \mathrm{Mn}$, UFeMn, and $\mathrm{UMn}_{2}$ show similar features. The DOS for these alloy systems contains a $5 f$ band, which is pinned at $E_{F}$ and strongly hybridizing with the iron and manganese $3 d$ states. The main weight of the $3 d$ states is positioned at about $2-3 \mathrm{eV}$ below $E_{F}$. The substitution of manganese with iron pushes the main weight of the $3 d$ band away from $E_{F}$ but leaves the overall features intact. It is, however, obvious from Fig. 13 that when comparing $\mathrm{UMn}_{2}$ with $\mathrm{UFe}_{2}$ in detail, one notices some deviations from a rigid-band behavior and therefore one should be somewhat cautious using a rigid-band model.

$$
\text { D. } \mathbf{U}\left(\mathrm{Fe}_{1-x} \mathrm{Cr}_{x}\right)_{2}
$$

The $\mathrm{UCr}_{2}$ compound has not been observed experimentally. However in alloys with $\mathrm{UFe}_{2}$ the pseudobinary $\mathrm{U}\left(\mathrm{Fe}_{1-x} \mathrm{Cr}_{x}\right)_{2}$ alloy system can be prepared over a rather large concentration range, extending up to $x=0.6 .^{1}$ Magnetic measurements on this system show that a transition from ferromagnetism to paramagnetism takes place when $x \approx 0.12 .^{1}$ This means that the ferromagnetism of $\mathrm{UFe}_{2}$ is severely suppressed by substituting $\mathrm{Fe}$ with $\mathrm{Cr}$. This is an even stronger reduction than for the substitution of iron with manganese (see above), but correlates well with the fact that chromium has about one $3 d$ elec- tron less than manganese. In fact the effective number of $3 d$ electrons corresponding to the critical concentration for the onset of magnetism in these two pseudobinary systems are almost identical. Similarly to the calculations above for $\mathrm{U}\left(\mathrm{Fe}_{1-x} \mathrm{Mn}_{x}\right)_{2}$ we considered the ordered system $\mathrm{U}_{2}\left(\mathrm{Fe}_{3} \mathrm{Cr}\right)$ and computed its Stoner product. From the interpolation shown as a dashed line in Fig. 12 we obtain that the magnetism should disappear at $x=0.15$. Here as well as in the manganese substituted alloys a rigid-band model of the DOS seems to be valid, and this is illustrated in the lower part of Fig. 13, where the DOS of $\mathrm{U}_{2} \mathrm{Fe}_{3} \mathrm{Cr}$ is plotted, again showing rather similar features as the DOS for $\mathrm{UFe}_{2}$, but with a shifted Fermi

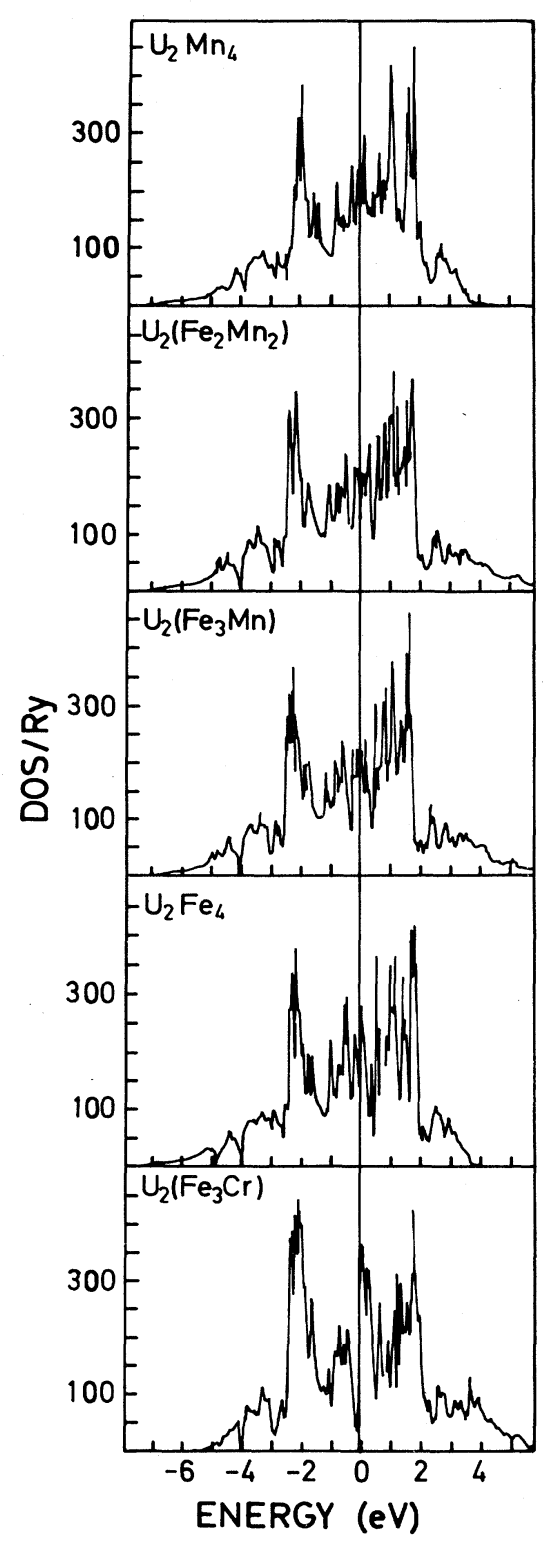

FIG. 13. Total DOS for $\mathrm{U}_{2} \mathrm{Fe}_{4}, \mathrm{U}_{2} \mathrm{Fe}_{3} \mathrm{Mn}, \mathrm{U}_{2} \mathrm{Fe}_{2} \mathrm{Mn}_{2}, \mathrm{UMn}_{4}$, and $\mathrm{U}_{2} \mathrm{Fe}_{3} \mathrm{Cr}$. Energies are in $\mathrm{eV}$ and zero is at the Fermi level. 
level. It should of course be noticed that the rigid band here only concerns the $3 d$ states. The U $5 f$ DOS remains relatively intact and stays pinned at the Fermi level in such a way that its occupation number is kept almost constant $\left(n_{f} \approx 2.7\right)$. In this sense the $5 f$ states are also rigid in the present pseudobinary alloys.

\section{CONCLUSION}

The multitude of the experimental data for the $\mathrm{Y}\left(\mathrm{Fe}_{1-x} \mathrm{Co}_{x}\right)_{2}$ pseudobinary system makes it very attractive to study theoretically. The properties of the two compounds $\mathrm{YFe}_{2}$ and $\mathrm{YCo}_{2}$, being a ferromagnet and an exchange-enhanced paramagnet, respectively, ensure that the pseudobinary alloy system is of particular interest for the study of the onset of magnetism in a random alloy. In the present work we have emphasized the variation of the magnetic moment with the alloy concentration and its direct influence on the equilibrium volume of the system. By means of a fixed-spin-moment calculation we found evidence that the disappearance of magnetism with alloy concentration is of first order. In connection with the calculations of the electronic structure of the $\mathrm{Y}\left(\mathrm{Fe}_{1-x} \mathrm{Co}_{x}\right)_{2}$ systems we also made an explicit comparison between a VCA and a supercell calculation. The difference was found to be small for $\mathrm{Y}\left(\mathrm{Fe}_{0.75} \mathrm{Co}_{0.25}\right)_{2}$. A similar check was done on UFeNi and its virtual crystal $\mathrm{UCo}_{2}$. Here we found that a supercell type of calculation gives a better description of the chemical bonding, whereas the state densities of the two approximations compare quite well. Concerning the magnetism in the pseudobinary $\mathrm{U}\left(\mathrm{Fe}_{1-x} \mathrm{Mn}_{x}\right)_{2}$ and $\mathrm{U}\left(\mathrm{Fe}_{1-x} \mathrm{Cr}_{x}\right)_{2}$ compounds we find that the Stoner product in $\mathrm{U}\left(\mathrm{Fe}_{1-x} \mathrm{Cr}_{x}\right)_{2}$ decreases twice as fast with $x$ as it does for $\mathrm{U}\left(\mathrm{Fe}_{1-x} \mathrm{Mn}_{x}\right)_{2}$. This can also be understood from a rigid-band model where the substitution of iron by another $3 d$ element merely shifts $E_{F}$ according the the number of valence electrons. Since chromium has two valence electrons less than iron, whereas manganese has one less, the substitution of iron with chromium shifts $E_{F}$ twice as fast with $x$ as a substitution with manganese would do, and correspondingly the Stoner product decreases twice as fast with $x$. This suggests that the magnetism in $\mathrm{UFe}_{2}$ is caused by the $3 d$ electrons and that the $5 f$ electrons actually play a parasitic role.
For these systems the validity of the rigid-band approximation is found to be good, although we emphasize that the description of an alloy system by studying an ordered ternary compound is in certain aspects more appropriate than the simple rigid-band model, since it maintains the individual properties of the atoms. However, since the shape and extension of the $3 d$ wave function of iron, manganese, and chromium in these types of pseudobinary alloys are very similar, this favors the validity of the rigid-band model. For instance, the paramagnetic ground state of the pseudobinary alloy $\mathrm{U}\left(\mathrm{Fe}_{0.5} \mathrm{Ni}_{0.5}\right)_{2}$ can be explained by a rigid-band model since this system has exactly the same number of valence electrons as the paramagnetic compound $\mathrm{UCO}_{2}$. However our analysis of this system reveals that this model, in common with the virtual-crystal approximation, in some aspects fails to describe its properties. The disadvantage of the present cluster approach is that although it treats both the concentration and coordination of the constituents of the alloy properly, it does not account for the random occupation of the $B$ sites in the $A B_{2}$ Laves system.

From the present $a b$ initio, self-consistent bandstructure calculations we have found that for the yttrium (uranium) $3 d$ Laves-phase pseudobinary alloys the treatment of the $3 d$ and $5 f$ electrons as delocalized is appropriate, since calculated detailed ground-state properties agree well with experimental data. This also means that the simulation of the electronic structure of a disordered alloy by that of an ordered compound with the same stoichiometry is a good approximation for the present systems. Hence it seems that the influence of disorder in the $3 d$ metal sublattice is of relatively minor importance.

\section{ACKNOWLEDGMENTS}

One of us (O.E.) is grateful to the Bank of Sweden Tercentenary Foundation and Börje Johansson is grateful to the Swedish Natural Research Council for financial support. Valuable discussions with Professor K. Schwarz and Dr. P. Mohn concerning the FSM calculations are acknowledged. Informative discussions with Dr. V. Sechovský and Dr. L. Havela concerning some of the experimental background of this work are also acknowledged.

\footnotetext{
*Present address: Center for Materials Science, Los Alamos National Laboratory, Los Alamos, New Mexico 87545.

${ }^{1}$ For instance, see V. Sechovský and L. Havela, in Intermetallic Compounds of Actinides, Vol. 4 of The Handbook of Ferromagnetic Materials, edited by E. P. Wohlfarth and K. H. J. Buschow (North-Holland, Amsterdam, 1988).

${ }^{2}$ V. Sechovský and G. Hilscher, Physica B + C 130B, 207 (1985); J. M. Fournier and R. Troć, in Handbook on the Physics and Chemistry of the Actinides, edited by A. J. Freeman and G. H. Lander (North-Holland, Amsterdam, 1985); Vol. 2, p. 29.

${ }^{3}$ O. Eriksson, B. Johansson, H. L. Skriver, and M. S. S. Brooks, Physica B + C 144B, 32 (1986); M. S. S. Brooks, O. Eriksson, and B. Johansson, Phys. Scr. 35, 52 (1987).
}

${ }^{4}$ G. Hilscher, J. Magn. Mater 25, 229 (1982); 27, 1 (1982).

${ }^{5}$ M. Cryot, D. Gignoux, F. Givord, and M. Lavagna, J. Phys. (Paris) Colloq. 40, C5-171 (1979); K. Schwarz and P. Mohn, J. Phys. F 14, L129 (1984); M. Yamada and M. Shimizu, ibid. 15, L175 (1985).

${ }^{6}$ H. Yamada, J. Inoue, K. Terao, S. Kanda, and M. Shimizu, J. Phys. F 14, 1943 (1984); P. Mohn and K. Schwarz, Physica B + C 130B, 26 (1984).

${ }^{7}$ K. H. J. Bushow and R. P. van Stapele, J. Appl. Phys. 41, 4066 (1970).

${ }^{8}$ G. Hilscher and V. Sechovský, Solid State Commun. 40, 881 (1981); V. Sechovský, Z. Smetana, G. Hilscher, E. Graz, and H. Sassik, Physica B + C 102B, 277 (1980). 
${ }^{9}$ P. H. Frings, thesis, University of Amsterdam (1984); J. J. M. Franse, P. H. Frings, F. R. de Boer, and A. Menovsky, in Physics of Solids Under High Pressure, edited by J. S. Shilling and R. N. Shelton (North-Holland, Amsterdam, 1981).

${ }^{10}$ J. R. Naegele, L. Manes, J. C. Spirlet, L. Pellegrini, and J. M. Fournier, Physica 102B, 122 (1980); J. R. Naegele, Physica B+C 130B, 52 (1985); J. Ghisen, R. L. Johnson, J. C. Spirlet, and J. J. M. Franse, J. Electron Spectrosc. Relat. Phenom. 37, 163 (1985); M. Hoechst, K. Tan, and U. Hillebrecht, J. Vac. Sci. Technol. A 4, 1585 (1986); J. R. Naegele, J. Ghisen, R. L. Johnson, and V. Sechovský, Phys. Scr. 35, 877 (1987).

${ }^{11}$ O. K. Andersen, Phys. Rev. B 12, 3060 (1975); H. L. Skriver, The LMTO Method (Springer, Berlin, 1984).

${ }^{12}$ O. Jepsen and O. K. Andersen, Solid State Commun. 9, 1763 (1971).

${ }^{13}$ U. von Barth and L. Hedin, J. Phys. C 5, 1629 (1972).

${ }^{14}$ P. Vargas and N. E. Christensen, Phys. Rev. B 35, 1993 (1987); V. P. Zhukov, A. Gubanov, O. Jepsen, N. E. Christensen, and O. K. Andersen, Philos. Mag. B 58, 139 (1988); J. W. D. Connolly and A. R. Williams, in The Electronic Structure of Complex Systems, NATO Advanced Study Institute, edited by P. Phariseau and W. M. Temmerman (Plenum, New York, 1984).

${ }^{15}$ O. Eriksson, B. Johansson and M. S. S. Brooks, J. Phys. (Paris) Colloq. 49, C8-295 (1988).

${ }^{16}$ B. Spunzar and B. Kozarzewski, Acta Phys. Pol. 1, 125 (1977); Phys. Status Solidi B 82, 205 (1977); B. Spunzar and P. A. Lindgard, Ris $\varnothing$ 350, 34 (1976).

${ }^{17}$ A. R. Piercy and K. N. R. Taylor, J. Phys. C 7, 1112 (1968); A. P. Guimares and D. St. P. Bunbury, J. Phys. F 3, 885 (1973); W. Steiner and H. Ortbauer, Phys. Status Solidi A 26, 451 (1974).

${ }^{18}$ S. H. Vosko and J. P. Perdew, Can. J. Phys. 53, 1385 (1975); J. F. Janak, Phys. Rev. B 16, 255 (1977).

${ }^{19}$ A. R. Williams, V. L. Moruzzi, J. Kübler, and K. Schwarz, Bull. Am. Phys. Soc. 29, 278 (1984).
${ }^{20}$ V. L. Moruzzi, J. F. Janak, and A. R. Williams, Calculated Electronic Properties of Metals (Pergamon, New York, 1978).

${ }^{21}$ D. G. Pettifor, Commun. Phys. 1, 1 (1976); A. R. Mackintosh and O. K. Andersen, in Electrons at the Fermi Surface, edited by $M$. Springford (Cambridge University Press, Cambridge, England, 1979); O. K. Andersen, O. Jepsen, and D. Glötzel, in Highlights in Condensed Matter Theory, edited by F. Bassani, F. Fumi, and M. Tosi (North-Holland, New York, 1985); M. S. S. Brooks, B. Johansson, and H. L. Skriver, in Handbook on the Physics and Chemistry of the Actinides, edited by A. J. Freeman and G. H. Lander (North-Holland, Amsterdam, 1984), Vol. 1, p. 153.

${ }^{22}$ B. Johansson and O. Eriksson (unpublished).

${ }^{23}$ L. Vegard, Z. Phys. 5, 17 (1921)

${ }^{24}$ R. C. Mansey, G. V. Raynor, and I. R. Harris, J. LessCommon Metals 14, 329 (1968).

${ }^{25}$ See, for instance, J. M. Ziman, Models of Disorder (Cambridge University Press, Cambridge, 1979).

${ }^{26}$ Actually, since both $\mathrm{UFe}_{2}$ and $\mathrm{UNi}_{2}$ are ferromagnetic and $\mathrm{U}\left(\mathrm{Fe}_{0.5} \mathrm{Co}_{0.5}\right)_{2}$ is paramagnetic it is not straightforward to apply Vegard's law properly. For $\mathrm{UNi}_{2}$ the magnetovolume effect will be very small, but for $\mathrm{UFe}_{2}$ it is not negligible. Subtracting from the experimental value for $\mathrm{UFe}_{2}$ the calculated magnetovolume effect (cf. Fig. 9) and then applying Vegard's law shows that the experimental value for $\mathrm{U}\left(\mathrm{Fe}_{0.5} \mathrm{Ni}_{0.5}\right)_{2}$ lies above the so obtained curve. Note that this is the same type of deviation as we find from the calculated data (Fig. 9). The main point, however, is that the data points for $\mathrm{UCo}_{2}$ are far below those for $\mathrm{U}\left(\mathrm{Fe}_{0.5} \mathrm{Co}_{0.5}\right)_{2}$ (experiment) and $\mathrm{UFeNi}$ (theory).

${ }^{27}$ M. S. S. Brooks and P. J. Kelly, Phys. Rev. Lett. 51, 1708 (1983); J. Sticht and J. Kübler, Solid State Commun. 54, 389 (1985); M. R. Norman and D. D. Koelling, Phys. Rev. B 33, 3803 (1986); M. S. S. Brooks, O. Eriksson, B. Johansson, J. J. M. Franse, and P. M. Frings, J. Phys. F 18, L33 (1988). 